\title{
Extranodal Marginal Zone B-Cell Lymphoma of Mucosa-Associated Tissue Type Involving the Dura
}

\section{Joon Young Choi, MD \\ Ji Hwan Chung, MD ${ }^{1}$ \\ Young Jun Park, MD \\ Geun Yong Jung, MD 1 \\ Tae Wook Yoon, MD ${ }^{1}$ \\ Yoon Jung Kim, MD2 \\ Tae kyu Lim, MD ${ }^{1}$ \\ Bong Seog Kim, MD ${ }^{1}$ \\ Seung-Hyun Nam, MD}

Departments of ${ }^{1}$ Internal Medicine and ${ }^{2}$ Pathology,

Veterans Health Service Medical Center, Seoul, Korea
Primary central nervous system marginal zone B-cell lymphoma (MZBCL) is very rare, with only a few reported cases worldwide. It has an indolent disease course with high cure potential. We experienced a rare case of dural MZBCL of mucosa-associated lymphoid tissue (MALT) in a 69-year-old man who presented with headache. A magnetic resonance imaging scan of brain showed a $1.9 \times 3.6-\mathrm{cm}$-sized extra-axial mass with a broad based dural attachment to the anterosuperior aspect of the falx cerebri, radiographically consistent with meningioma. Surgical resection yielded a MZBCL of the MALT type. Histopathology revealed a lymphoplasmacytic infiltration of the dura, and immunohistochemical study showed a B-cell phenotype with CD20, bcl-2, MUM1, Ki-67 positive. He was treated with chemotherapy after complete surgical resection and remained free of disease at 30 months after chemotherapy. MALT Iymphoma must be considered in the differential diagnosis in patients presenting radiographically with meningioma.
Correspondence: Seung-Hyun Nam, MD Division of Oncology,

Department of Internal Medicine,

Veterans Health Service Medical Center,

53 Jinhwangdo-ro 61-gil, Gangdong-gu,

Seoul 05368, Korea

Tel: 82-2-2225-1669

Fax: 82-2-2225-4374

E-mail: mednsh@hanmail.net

Received November 24, 2014

Accepted April 29, 2015

Published Online July 17, 2015

\section{Key words}

Dura mater, Marginal zone B-cell lymphoma,

Central nervous system neoplasms

\section{Introduction}

Marginal zone B-cell lymphoma (MZBCL) is a generally low-grade, indolent lymphoma with a favorable response to treatment [1]. MZBCL, first described as a distinct low-grade lymphoma of mucosa-associated lymphoid tissue (MALT) of the gastrointestinal tract, has since been described in many other organs, including the lung, bladder, salivary glands, conjunctiva, and lacrimal glands [2]. According to 2008
World Health Organization classification, MZBCL is a subtype of non-Hodgkin lymphoma (NHL) that includes three distinct diseases historically classified together because they appear to arise from post-germinal center marginal zone B cells and share a similar immunophenotype [3]. Extranodal MZBCL of MALT is defined as an extranodal lymphoma composed of morphologically heterogenous small B cells, including marginal cells, cell resembling monocytoid cells, small lymphocytes, scattered immunoblasts, and centroblastlike cells [4]. Although the gastrointestinal tract is the most 

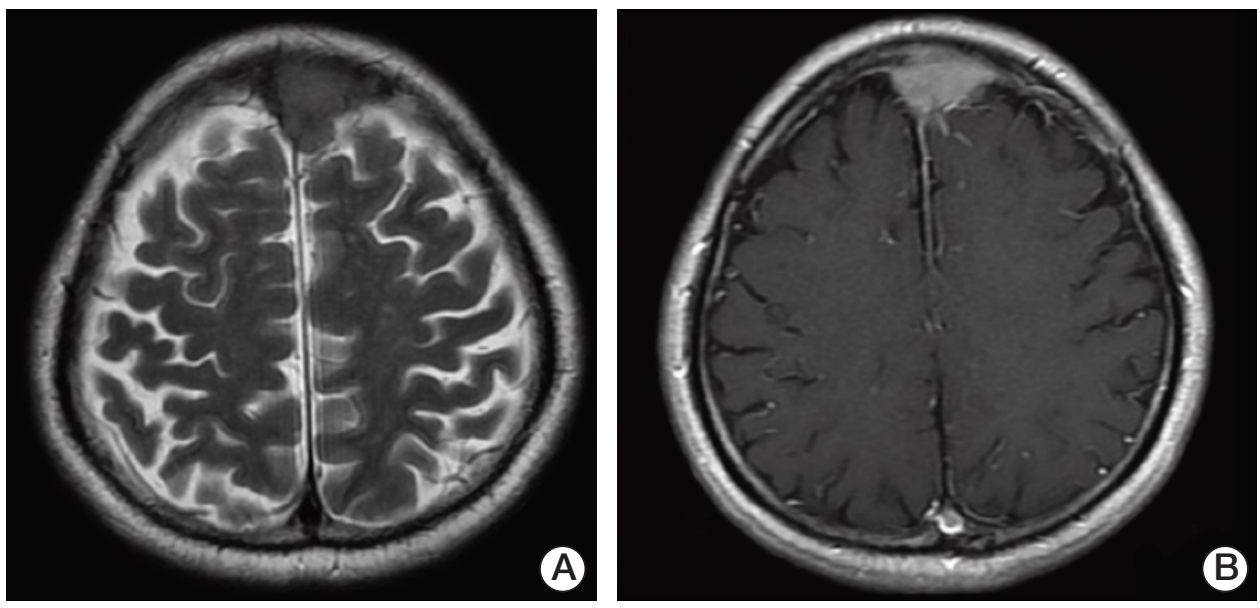

Fig. 1. Magnetic resonance imaging of the brain. (A) T2 weighted image shows the $1.9 \times 3.6$ extra-axial mass with broad based dural attachment to anterosuperior aspect of falx cerebri involving superior sagittal sinus. (B) T1 weighted image. The mass showed dural tail sign and a suspicious cerebrospinal fluid cleft sign.

common site of MALT lymphoma and these tumors are closely associated with mucosal tissue, MALT lymphoma may also present in organs and tissue sites without a mucosa, such as thyroid, breast, skin, liver, orbit, and thymus tissue [2].

Primary central nervous system (CNS) lymphoma is defined as a lymphoma with no evidence of disease outside the brain, spinal cord, and eye at the time of diagnosis. Primary CNS MZBCL is very rare and often misdiagnosed as meningioma, because its location and radiologic appearance mimic meningioma [1]. This primary lymphoma follows an indolent course, with a favorable response to treatment.

In the present report, we describe a patient with primary CNS MZBCL arising in the dura mimicking meningioma.

\section{Case Report}

A 69-year-old man visited at our hospital in January 2012 with a 2-month history of headache. The patient had no trauma history and no significant medical history. He had no other objective neurologic finding. A magnetic resonance imaging (MRI) scan of brain showed a relatively homogeneous enhancing $1.9 \times 3.6-\mathrm{cm}$-sized extra-axial mass with a broad based dural attachment to the anterosuperior aspect of the falx cerebri involving the superior sagittal sinus. The mass showed dural tail sign, and because of suspicious cerebrospinal fluid cleft sign, the mass was considered of dura origin (Fig. 1). He was diagnosed as meningioma radiograp- hically. He underwent bifrontal craniotomy with total removal of the tumor. The mass was completely resected with good dural margins. Pathologic examination revealed that the majority of the tumor cells were monocytoid B-cells, which have small to medium sized nuclei and abundant pale cytoplasm (Fig. 2). There were also remnants of reactive follicles and follicular colonization in the tumor. Immunophenotypically the tumor cells were positive for CD20, bcl-2, and MUM-1 but negative for CD3, CD5, CD10, cyclin D1, and bcl6. Ki-67 labeling index was 15\%. Overall, these features are compatible with extranodal MZBCL of MALT type.

The patient underwent chest computed tomography (CT), abdominal and pelvic $\mathrm{CT}$, spine MRI, bone marrow aspiration and biopsy, and cerebrospinal tapping for staging workups. These did not reveal any abno-rmalities. Ophthalmologic evaluations revealed no other areas of disease involvement. The laboratory finding was normal. Rapid urease test for Helicobacter pylori was negative. After the bifrontal craniotomy with total removal of the tumor, the patient was treated with systemic chemotherapy with cyclophosphamide, vincristine and prednisolone for six cycles without treatment-related complication. Follow-up positron emission tomographic scan showed him to be in disease-free. As of October 2014, our patient has been free of symptoms or recurrence for 2 years and 9 months. 

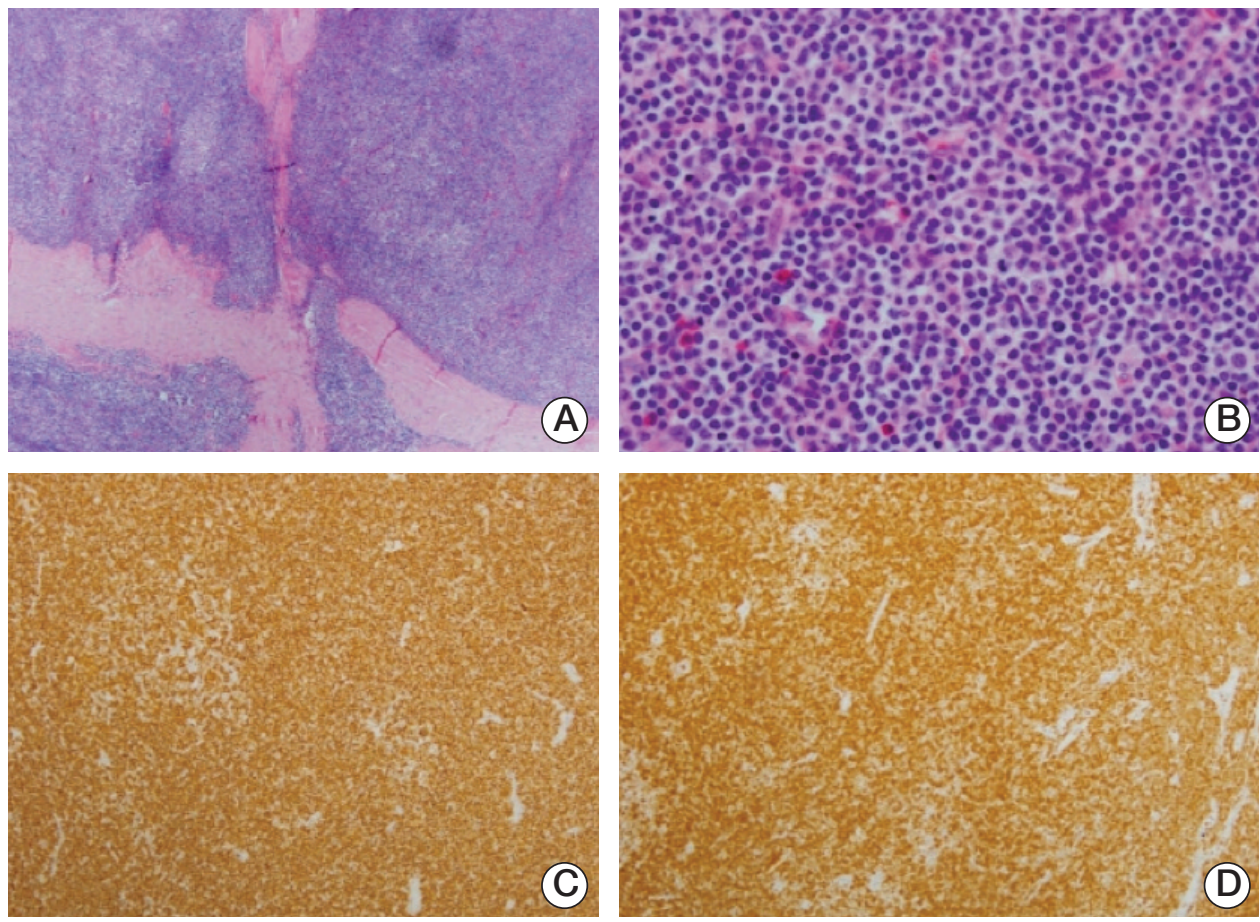

Fig. 2. Histological and immunohistochemical finding. (A) The central collagen band-like structure, dura mater and the tumor consisting of lymphoid tissue (H\&E staining, $\times 40$ ). (B) The tumor is mainly composed of monocytoid B cells having small to medium sized nuclei and abundant pale cytoplasm (H\&E staining, $\times 400)$. (C, D) The tumor cells show immunoreactivity for CD20 (C) and bcl-2 (D) (×100).

\section{Discussion}

MZBCL is a type of B-cell lymphoma presenting primarily in the marginal zone. Extranodal MZBCL of MALT is defined as an extranodal lymphoma, and MALT lymphomas typically present as a local mass without bone marrow and lymph node involvement, in contrast to nodal and splenic MZBCL [5]. The stomach and ocular adnexa are the most common sites for the MALT lymphomas. It is also recognized in intestinal tract, lung, thyroid, salivary glands, skin, liver, CNS, and breast [2].

Primary CNS lymphoma constitutes only $3 \%$ of primary brain tumors [6]. The majority of primary CNS lymphoma are diffuse large B-cell lymphoma that are mostly intraparenchymal [7]. T-Cell, low-grade B-cell lymphoma and anaplastic large cell lymphoma of the CNS are extremely rare [7]. Most primary CNS lymphoma presents as a space-occupying lesion within the brain parenchyma and periventricular regions [8]. Primary CNS MZBCL is very rare and indolent and occurs mostly in the extra-axial dura, in contrast to primary CNS high-grade lymphoma [1]. Cases of dural MZBCL in the literature are reviewed in Table 1.
We reported a case of a MALT-type lymphoma of the dura presenting symptomatically and radiographically as meningioma. Primary CNS MZBCL arises mostly from areas rich in meningothelial cells or throughout the arachnoid membrane and arachnoid villi within the dural venous sinus [1]. Because meningiomas are also common to these sites, dural MALT lymphoma has often been misdiagnosed as meningioma. CT or MRI findings reveal well defined, intracranial, dural mass. Presenting symptoms vary by tumor location; patients usually present with headache, meningeal signs, and cranial nerve involvement $[8,9]$.

The diagnosis in our case was confirmed by the histological and immunophenotyping findings, but CNS MALT lymphoma can be difficult to identify even with immunophenotyping. In our case, immunohistochemical stains were negative for CD3, CD5, CD10, while CD20 was positive.

There is no defined treatment guideline for this rare clinical entity. Primary CNS MALT lymphoma originating from the dura matter is curable by surgical removal and radiation therapy compared to parenchymal primary CNS lymphoma or systemic lymphoma with CNS metastasis [9]. Although no standard of care is established, it would seem reasonable 
Table 1. Summary of patient characteristics with CNS dura involvement by MZBCL

\begin{tabular}{lcccccc|} 
Case reference & Sex & Age (yr) & Location & Treatment & Status & Follow-up (mo) \\
\hline Tu et al. [11] & M & 49 & Frontal & CTx & CR1 & 90 \\
& M & 66 & Frontal & RTx & CR1 & 13 \\
\hline Iwamoto et al. [12] & F & 64 & Temporal & Resection+CTx & CR1 & 78 \\
& F & 33 & Frontal & CTx+RTx & CR1 & 84 \\
\hline Kumar et al. [13] & F & 52 & Frontal & CTx+RTx & CR1 & 7 \\
& F & 43 & Tentorium & RTx & CR1 & 9 \\
\hline Sanjeevi et al. [14] & F & 46 & Cavernous sinus & Resection+RTx & CR1 & 15 \\
Lehman et al. [15] & F & 63 & Falx cerebri & RTx & AWD & 8 \\
Kamoshima et al. [9] & M & 55 & Frontal & RTx & CR1 & 36 \\
\hline
\end{tabular}

CNS, central nervous system; MZBCL, marginal zone B-cell lymphoma; M, male; CTx, chemotherapy; RTx, radiotherapy; F, female; AWD, alive with disease.

to treat with aggressive radiotherapy, with or without surgical resection, depending on the amount of mass [10]. The radiation field is determined by the extent of the dural lesions and leptomeningeal involvement. Therefore, this rare subtype is important to distinguish from other ordinary B-cell NHL with poor clinical outcomes. In some cases, chemotherapy might be suitable as the sole adjuvant treatment. The dura mater is outside the blood-brain barrier, which may explain the increased risk of systemic relapse compare to other types of primary CNS lymphoma. In our case, cyclophosphamide, vincristine and prednisolone chemotherapy brought about a disease-free state.
In summary, we have presented a very rare case of primary CNS MZBCL involving the dura. Longer clinical observation can help to assess the final outcome of patients with primary CNS MALT lymphoma.

\section{Conflicts of Interest}

Conflict of interest relevant to this article was not reported.

\section{References}

1. Park I, Huh J, Kim JH, Lee SW, Ryu MH, Kang YK. Primary central nervous system marginal zone B-cell lymphoma of the Basal Ganglia mimicking low-grade glioma: a case report and review of the literature. Clin Lymphoma Myeloma. 2008;8: 305-8.

2. Bayraktar S, Stefanovic A, Montague N, Davis J, Murray T, Lossos IS. Central nervous system manifestations of marginal zone B-cell lymphoma. Ann Hematol. 2010;89:1003-9.

3. Jaffe ES. The 2008 WHO classification of lymphomas: implications for clinical practice and translational research. Hematology Am Soc Hematol Educ Program. 2009:523-31.

4. Terada T. Extranodal marginal zone B-cell lymphoma of mucosa-associated lymphoid tissue (MALT lymphoma) in ulcerative colitis. Saudi J Gastroenterol. 2014;20:319-22.

5. Bhagavathi S, Greiner TC, Kazmi SA, Fu K, Sanger WG, Chan WC. Extranodal marginal zone lymphoma of the dura mater with IgH/MALT1 translocation and review of literature. J Hematop. 2008;1:131-7.

6. Abbi KK, Muzaffar M, Gaudin D, Booth RL Jr, Feldmeier JJ,
Skeel RT. Primary CNS lymphoplasmacytic lymphoma: a case report and review of literature. Hematol Oncol Stem Cell Ther. 2013;6:76-8.

7. Giannini C, Dogan A, Salomao DR. CNS lymphoma: a practical diagnostic approach. J Neuropathol Exp Neurol. 2014;73: 478-94.

8. Sacho RH, Kogels M, du Plessis D, Jowitt S, Josan VA. Primary diffuse large B-cell central nervous system lymphoma presenting as an acute space-occupying subdural mass. J Neurosurg. 2010;113:384-7.

9. Kamoshima Y, Sawamura Y, Sugiyama T, Yamaguchi S, Houkin K, Kubota K. Primary central nervous system mucosaassociated lymphoid tissue lymphoma: case report. Neurol Med Chir (Tokyo). 2011;51:527-30.

10. Shaia J, Kerr PB, Saini A, Roberti F, Kapil J, Jones R, et al. Mucosa-associated lymphoma tissue of the dura presenting as meningioma. South Med J. 2010;103:950-2.

11. Tu PH, Giannini C, Judkins AR, Schwalb JM, Burack R, O'Neill $\mathrm{BP}$, et al. Clinicopathologic and genetic profile of intracranial 
marginal zone lymphoma: a primary low-grade CNS lymphoma that mimics meningioma. J Clin Oncol. 2005;23: 5718-27.

12. Iwamoto FM, DeAngelis LM, Abrey LE. Primary dural lymphomas: a clinicopathologic study of treatment and outcome in eight patients. Neurology. 2006;66:1763-5.

13. Kumar S, Kumar D, Kaldjian EP, Bauserman S, Raffeld M, Jaffe ES. Primary low-grade B-cell lymphoma of the dura: a mucosa associated lymphoid tissue-type lymphoma. Am J
Surg Pathol. 1997;21:81-7.

14. Sanjeevi A, Krishnan J, Bailey PR, Catlett J. Extranodal marginal zone B-cell lymphoma of malt type involving the cavernous sinus. Leuk Lymphoma. 2001;42:1133-7.

15. Lehman NL, Horoupian DS, Warnke RA, Sundram UN, Peterson K, Harsh GR 4th. Dural marginal zone lymphoma with massive amyloid deposition: rare low-grade primary central nervous system B-cell lymphoma. Case report. J Neurosurg. 2002;96:368-72. 